\title{
Anatomo-radiological correlation using 18-FDG-PET in abdominal sepsis model in rats. A preliminary study ${ }^{1}$
}

Ítalo Medeiros Azevedo', Marília Daniela Ferreira Carvalho', Rafael Pereira Nascimento", Robson Macedo'", Mônica Raquel de Souza Aquino'v, Aldo Cunha Medeirosv

'Fellow PhD degree, Postgraduate Program in Health Sciences, Universidade Federal do Rio Grande do Norte (UFRN), NatalRN, Brazil. Acquisition and interpretation of data, technical procedures, manuscript preparation.

"Fellow Master degree, Postgraduate Program in Health Sciences, UFRN, Natal-RN, Brazil. Acquisition and interpretation of data, technical procedures.

I'PhD, Radiology Institute of Natal, Brazil. Analysis and interpretation of data, technical procedures, critical revision.

IVGraduate student, Institutional Program of Scientifical Initiation, UFRN, Natal-RN, Brazil. Acquisition of data, technical procedures, manuscript preparation.

${ }^{\vee}$ Full Professor, Chairman, Nucleus of Experimental Surgery, UFRN, Natal-RN, Brazil. CNPq/PQ.2 fellowship. Conception, design, intellectual and scientific content of the study; analysis and interpretation of data; critical revision.

\section{Abstract}

Purpose: To examine a correlation of micro-PET images with photographic images of the digestive organs in abdominal sepsis model.

Methods: Male Wistar rats weighing $265 \pm 18 \mathrm{~g}$ were used. Abdominal sepsis was induced by ligature and cecal puncture. Micro-PET Images from abdominal cavity septic foci were obtained using 18-Fluoro-deoxyglucose, looking for a correlation with photographic images of abdominal cavity organs. Pearson's correlation test was used.

Results: The mean standard uptake values (SUV) and lesion areas were $2.58 \pm 0.63 \mathrm{SUVbwg} / \mathrm{ml}$ and $546.87 \pm 300.95 \mathrm{~mm}^{2}$, respectively. There was a strong positive correlation between the two variables $(r=0.863, p=0.137)$, which resulted in a coefficient of determination $r^{2} \cong 0.75$, meaning that $75 \%$ of SUV variation is explained by the lesion areas of digestive organs.

Conclusion: Micro-PET allows high throughput assessment of lesion count and volume in pre-clinical rat model of CPL abdominal sepsis..

Key words: Sepsis. Fluorodeoxyglucose F18. Positron-Emission Tomography. Rats. 


\section{- Introduction}

One of the major current public health problems remains sepsis, which persists with high hospital mortality. In sepsis, the infection almost always occurs, resulting in eventual severe systemic response, classified in several stages $^{1}$. Because of its seriousness and rapid evolution, it is important to make quick and accurate decisions to diagnose sepsis, more specifically abdominal sepsis.

Currently, the diagnostic means necessary for a specific and accurate clinical evaluation to clarify whether or not the patients have sepsis has been insufficient in many cases $^{2}$. Establishing an order of effectiveness and complexity, conventional radiography, ultrasonography, computed tomography (CT) and nuclear magnetic resonance imaging (MRI) have been the most widely used imaging tests to attempt the diagnosis of abdominal sepsis. Conventional radiography was very useful in the years 1970-90, and continues with high percentages of false positive and false negative results ${ }^{2,3}$. Ultrasonography is very affordable in most hospitals and communities, does not have the inherent risks of imaging tests that use $x$-rays, and since it does not include the use of contrasts, the toxicity of the test is almost nil ${ }^{6,7}$. CT, when compared to conventional radiography and ultrasonography, has greater sensitivity and specificity. In cases of appendicitis and diverticulitis, it has been shown that MRI is accurate in its diagnosis ${ }^{8,9}$. In addition, MRI, like ultrasonography, does not include the use of contrast media and does not expose patients to $x$-ray ${ }^{10}$.

18F-fluorodeoxyglucose (18F-FDG) was initially developed for oncology, specifically for staging and follow-up of cancer patients. 18F-FDG accumulate in infected areas due to high uptake of glucose and its use by leukocytes. FDG-PET has been shown to be useful in the diagnosis of fever of unknown origin. As it is a tomography, it is an examination of choice by the anatomical details that it is able to demonstrate in cross-sections ${ }^{11-13}$. In cases of infection, FDG-PET has been useful in the diagnosis of osteomyelitis, spondylitis, infected liver cysts, and other infectious diseases ${ }^{14,15}$. However, FDG-PET loses specificity because it can enhance any area of high glycolytic activity including trauma, wound, cancer, etc. Currently, the great challenge with FDG-PET has been its high cost. Finally, we do not know of previous studies that evaluated the validity of 18-FDG-PET in animal models of sepsis.

The objective of this preliminary study is to examine the correlation between tomographic images by 18-FDG-PET and photographic images in abdominal sepsis model in rats.

\section{- Methods}

The protocol was approved by the institutional Ethics Committee on Animal Use (protocol no 003/2015). All experimental procedures were performed based on the guidelines of Brazilian Law No. 11.794 (CONCEA).

\section{Experimental design}

Male Wistar rats weighing $265 \pm 18 \mathrm{~g}$ were used. Four animals were submitted to abdominal sepsis by ligature and cecal puncture and, 24 hours later, were examined through Positron Emission Tomography and photographic images of the open abdominal cavity.

\section{Sepsis induction}

The rats $(n=4)$ were anesthetized with xylazine associated with ketamine at doses of 10 and $70 \mathrm{mg} / \mathrm{kg}$, respectively, intramuscular. The induction of sepsis by cecal ligation and puncture (CLP) followed the technique used in other studies $^{16-19}$. Briefly, after shaving and disinfecting the lower quadrants of the abdomen with 
alcohol $70 \%$, a midline laparotomy of $4 \mathrm{~cm}$ length was performed. A cecal ligation with silk 000 was positioned at the distal third of the cecum. Two perforation of the cecum was performed using a $18 \mathrm{G}$ needle. A gently pressure was performed to permit small amount of fecal material in both perforation holes. After repositioning the cecum within the abdominal cavity, the abdomen was sutured in two layers with nylon 4-0 suture. Postoperative pain was controlled with tramadol $10 \mathrm{mg} / \mathrm{kg}$ s.c. immediately after surgery and every 12 hours.

Imaging with 18-FDG-PETscan in rats

2-[18F]fluoro-2-deoxy-D-glucose (18F-FDG) was purchased from Vilas Boas Radiofármacos (Brasilia-DF, Brazil). Completed 24 hours post-induction of sepsis, rats were anesthetized by the same technique described above. Right femoral vein was dissected for injection. 18F-FDG (22 MBq) $0.2 \mathrm{ml}$ was injected i.v. in each animal, and the dynamic image acquisition started after $\mathbf{4 0}$ minutes. The three-dimensional images of Positrion Emission Tomography (PET) were taken in the whole body of rats, using the Preclinical Imaging Albira PET (Bruker, New Haven, CT, USA).

The standard uptake values (SUV) of the tracer represented in PET images were quantified using the PMOD software, considering the organs and regions of interest (ROI) of abdominal cavity under study.

\section{Photographic examination of the abdomen}

With animals still anesthetized, euthanasia was performed with an overdose of sodium thiopental $(100 \mathrm{mg} / \mathrm{kg}$ ) via intracardiac injection. A large midline laparotomy was performed, for access to the abdominal organs, looking for septic foci and other findings. The abdomen was photographed using a digital camera Sony DSC-HX100V, 16.2 mega pixels, Japan. Quantitative study of lesions areas was performed using Media Cyber ProPlus 5.0 software. Measurement of photographic images were stored for anatomo-radiologic correlation with SUV of micro-PET images.

\section{Statistical analysis}

Quantitative variables - area of injury and SUV - were analyzed using Pearson's correlation test, with significance level of $5 \%$ using the $R$ software ${ }^{20}$.

\section{- Results}

Septic lesions documented on microPET and photo images were analyzed. From each rat, the abdominal organs were imaged using photographic camera, micro-PET coronal image and micro-PET axial image (Figure 1). Moreover, a robust correlation was observed between the lesional cecal areas documented by photo images quantified through ImagePro plus software and the SUV, using the microPET scan approach. Additionally, inflamedhyperemic intestinal loops were identified.

The mean SUV values and lesion areas were $2.58 \pm 0.63 \mathrm{SUVbw} \mathrm{g} / \mathrm{ml}$ and $546.87 \pm 300.95 \mathrm{~mm} 2$, respectively. There was a strong positive correlation between the two variables $(r=0.863, p=0.137)$, which resulted in a coefficient of determination $r 2 \cong 0.75$, meaning that $75 \%$ of SUV variation is explained by the lesion area (Pearson's correlation test). 

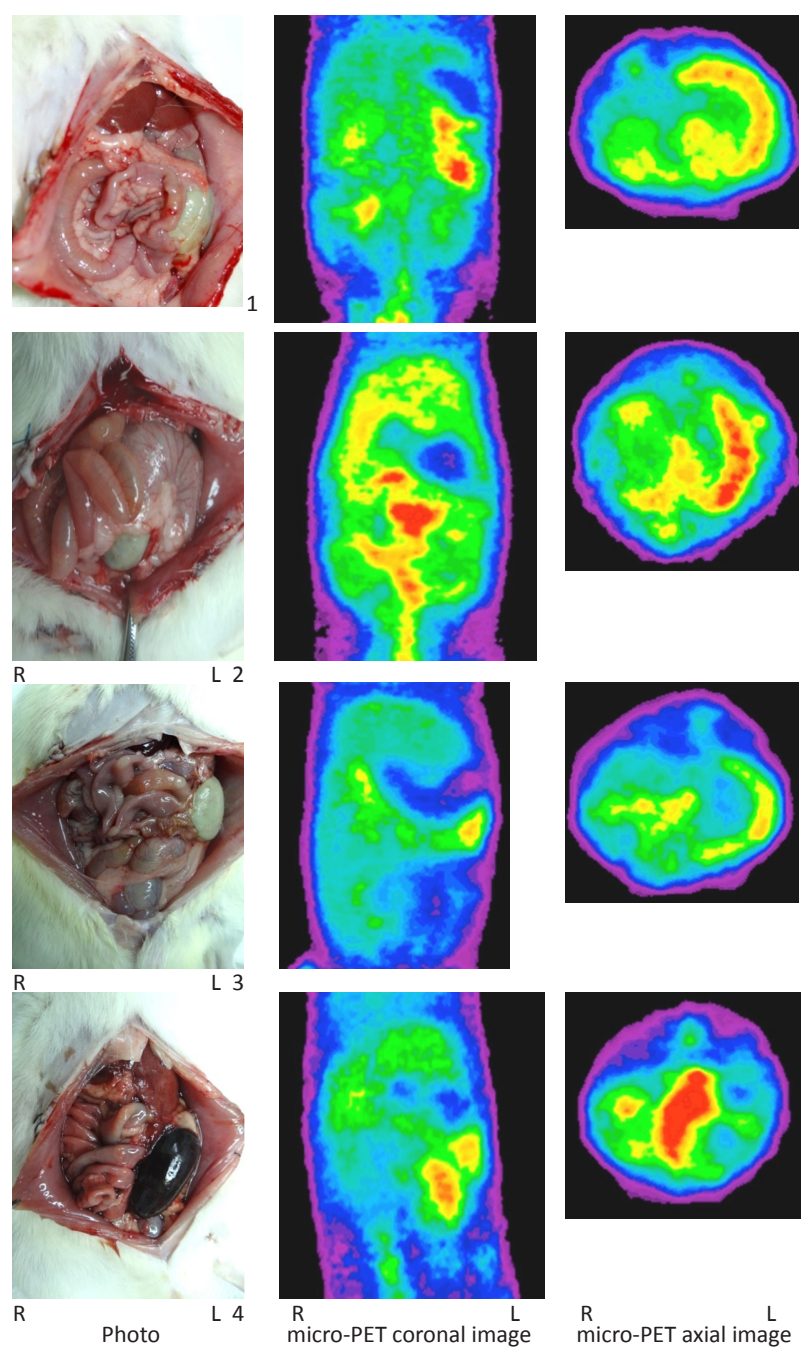

Figure 1 - From rats 1, 2, 3 and 4, abdominal organs images from photographic camera are correlated with micro-PET coronal and axial images ( $\mathbf{R}$, right; L, left).

\section{- Discussion}

Inflammatory and infectious processes are characterized byseveral pathophysiological mechanisms in which nuclear medicine examinations are based to turn them into diagnostic images. Radiopharmaceuticals are detected in the affected organs depending on these mechanisms, including leukocyte migration and increased vascular permeability in infectious foci $^{21}$. In the last three decades, imaging studies of conventional nuclear medicine were basically planar scintigraphy and scintigraphy using labeled leukocytes ${ }^{22,23}$. More recently, came the idea of using of positron emission tomography using fluor-18fluorodexoyglucose (18F-FDG)-PET for clinical diagnosis of infectious and inflammatory diseases ${ }^{24,25}$.

The use of experimental models of sepsis in rodents has given us the opportunity to better understand the pathogenesis, diagnosis and treatment of sepsis. Several parameters involving microbiology, molecular biology and immunogenetics have been employed in studies of abdominal sepsis. However, to quantify the lesions resulting from it, only the imaging tests can offer convincing data, especially when it comes to image coupled with molecular biology. Based on the assumption that there is limited knowledge about the diagnosis of abdominal sepsis with PET, in this study a preliminary study was conducted to correlate abdominal cavity anatomical images with micro PET images in an animal model of sepsis by CLP. Stimulated with the wide use of micro PET images in animal models of abdominal diseases, including tumors and infection, our purpose was to test and validate this technique in the murine preclinical model of abdominal sepsis. Our protocol was able to detect and localize septic lesions in the present model in rats, imitating lesions observed in humans.

Lesions identified on micro-PET were faced up to the photographic images demonstrating a high reliability in lesions identified by differentiating them from normal abdominal anatomy. Furthermore, the computer-based lesional standard uptake values (SUV) had a strong correlation with the integrated lesional areas assessed by the photographic images of the abdominal cavity immediately done after microPET-scan. The spatial resolution on the microPET-scan system was sufficient to correctly identify the abdominal organs, even the unaffected ones. This direct visualization of septic organs 
validated the accuracy of the protocol which was confirmed by satisfactory rates of visualization of the 18-FGD-PET signs. In the present study, a robust correlation was observed with the anatomical images.

To the best of our knowledge, this must have been the first time that a correlation between anatomical images and microPET-scan in abdominal sepsis model was studied in an experimental protocol. Our microPET-scan study allowed a radiological correlation method between the abdominal sepsis using CLP and anatomical reference photographs. Based on these results, future studies will be conducted in this research field. So, this protocol may be very helpful for our learning curve in micro-PET imaging, each time we need to assess septic focus in the abdominal cavity. This will be of great importance in future studies. Clinical benefits of this knowledge will certainly be greater for studing small septic lesions and fever of unknowing origin in future experimental protocols ${ }^{11}$. Considering the scarce number of publications on diagnosis of abdominal sepsis by 18 FDG-PET, and little experience with this imaging method, our results support its viability for abdominal sepsis. So, after a short learning curve, the analysis of the PETscan data may become a routine practice in cases of abdominal sepsis by radiological services.

A limitation of our microPET imaging is that despite a relatively high spatial resolution, the anatomic information available with our micro-PET was a little limited. To improve this, we are planning the acquisition of integrated in-line PET/CT system that must provide more accurate co-registered PET and CT images, as pointed for some authors ${ }^{26,27}$.

\section{Conclusion}

Micro-PET allows the evaluation of high yield by means of the quantification of damaged areas in the preclinical model of sepsis in rats. This assessment correlates accurately with anatomical lesion images and may be applied for future research.

\section{- References}

1. Levy MM, Fink MP, Marshall JC, Abraham E, Angus D, Cook D, Cohen J, Opal SM, Vincent JL, Ramsay G; SCCM/ESICM/ACCP/ ATS/SIS. 2001 SCCM/ESICM/ACCP/ATS/SIS International Sepsis Definitions Conference. Crit Care Med. 2003;31(4):1250-6. doi: 10.1097/01.CCM.0000050454.01978.3B.

2. Lameris W, van Randen $A$, van Es HW, van Heesewijk JP, van Ramshorst B, Bouma $\mathrm{WH}$, et al: Imaging strategies for detection of urgent conditions in patients with acute abdominal pain: diagnostic accuracy study. BMJ. 2009 Jun;338:b24-31. doi: 10.1136/ bmj.b2431.

3. van Randen A, Laméris $W$, Luitse JS, Gorzeman M, Hesselink EJ, Dolmans DE, Peringa J, van Geloven AA, Bossuyt PM, Stoker J, Boermeester MA; OPTIMA study group. The role of plain radiographs in patients with acute abdominal pain at the ED. Am J Emerg Med. 2011 Jul;29(6):582-9. doi: 10.1016/j.ajem.2009.12.020.

4. Lindelius A, Törngren S, Sondén A, Pettersson $H$, Adami J. Impact of surgeon-performed ultrasound on diagnosis of abdominal pain. Emerg Med J. 2008 Aug;25(8):486-91. doi: 10.1136/emj.2007.052142.

5. Nural MS, Ceyhan M, Baydin A, GencS, Bayrak IK, Elmali M. The role of ultrasonography in the diagnosis and management of nontraumatic acute abdominal pain. Intern Emerg Med. 2008 Dec;3(4):349-54. doi: 10.1007/s11739-008-0157-8.

6. Rudnick M, Feldman H. Contrast-induced nephropathy: what are the true clinical consequences? Clin J Am Soc Nephrol. 2008 Jan;3(1):263-72. doi: 10.2215/ CJN.03690907.

7. Rao QA, Newhouse JH. Risk of nephropathy after intravenous administration of contrast material: a critical literature analysis. Radiology. 2006 May;239(2):392-7. doi: 10.1148/radiol.2392050413.

8. Heverhagen JT, Zielke A, Ishaque N, Bohrer $\mathrm{T}$, El-Sheik M, Klose KJ. Acute colonic diverticulitis: visualization in magnetic resonance imaging. Magn Reson Imaging. 2001 Dec;19(10):1275-7. PMID: 11804754.

9. Leeuwenburgh MM, Wiarda BM, Wiezer MJ, 
Vrouenraets BC, Gratama JW, Spilt A, Richir MC, Bossuyt PM, Stoker J, Boermeester MA; OPTIMAP Study Group. Comparison of imaging strategies with conditional contrastenhanced CT and unenhanced MR imaging in patients suspected of having appendicitis: a multicenter diagnostic performance study. Radiology. 2013 Jul;268(1):135-43. doi: 10.1148/radiol.13121753.

10.Leeuwenburgh MM, Wiarda BM, Bipat S. Acute appendicitis on abdominal MR images: training readers to improve diagnostic accuracy. Radiology. 2012 Aug;264(2):45563. doi: 10.1148/radiol.12111896.

11. Castaigne C, Tondeur M, de Wit S, Hildebrand M, Clumeck N, Dusart M. Clinical value of FDG-PET/CT for the diagnosis of human immunodeficiency virus-associated fever of unknown origin: a retrospective study. Nucl Med Commun. 2009 Jan;30(1):41-7. PMID: 19306513.

12.Basu S, Chryssikos T, Moghadam-Kia S, Zhuang $\mathrm{H}$, Torigian DA, Alavi A. Positron emission tomography as a diagnostic tool in infection: Present role and future possibilities. Semin Nucl Med. 2009 Jan;39(1):36-51. PMID: 19038599.

13.Bleeker-Rovers CP, de Kleijn EM, Corstens $\mathrm{FH}$, van der Meer JW, Oyen WJ. Clinical value of FDG PET in patients with fever of unknown origin and patients suspected of focal infection or inflammation. Eur J Nucl Med Mol Imaging. 2004 Jan;31(1):29-37. PMID: 14551752.

14.Ito K, Kubota K, Morooka M, Hasuo K, Kuroki $\mathrm{H}$, Mimori A. Clinical impact of (18) FFDG PET/CT on the management and diagnosis of infectious spondylitis. Nucl Med Commun. 2010 Aug;31(8):691-8. doi: 10.1097/MNM.0b013e32833bb25d.

15. Bleeker-Rovers CP, de Sevaux RG, van Hamersvelt HW, Corstens FH, Oyen WJ. Diagnosis of renal and hepatic cyst infections by 18- F-fluorodeoxyglucose positron emission tomography in autosomal dominant polycystic kidney disease. Am J Kidney Dis. 2003 Jun;41(6):E18-21. PMID: 12776306.

16.Macedo R, Javadi SM, Higuchi T, Carvalho MD, Medeiros Vde F, Azevedo ÍM, Lima FP, Medeiros AC. Heart and systemic effects of statin pretreatment in a rat model of abdominal sepsis. Assessment by Tc99m- sestamibi biodistribition. Acta Cir Bras. 2015 Jun;30(6):388-93. doi: 10.1590/S0102865020150060000003.

17. Botelho NM, Silveira EL, Lopes LN, Santos FA, Teixeira RK, Silva TT. Copaiba oil effect under different pathways in mice subjected to sepsis. Acta Cir Bras. 2014 Aug;29(8):52831. PMID: 25140595.

18.Demir M, Taylan M, Kaya H, Ekinci A, Arslan D, Aslan E, Keles A, Yılmaz S, Sezgi C. Histopathological and biochemical effects of Ecballium elaterium on sepsis-induced lung injury. J Invest Surg. 2016 Oct;29(5):302-8. doi: 10.3109/08941939.2015.1111474.

19.Sozutek A, Colak T, Cetinkunar S, Reyhan E, Irkorucu O, Polat G, Cennet A. The effect of platelet-rich-plasma on the healing of left colonic anastomosis in a rat model of intra-abdominal sepsis. J Invest Surg. 2016 Oct;29(5):294-301. doi: 10.3109/08941939.2015.1111473.

20.R Development Core Team (2008) R Foundation for Statistical Computing, Vienna, Austria.

21.Goldsmith SJ, Vallabhajosula S. Clinically proven radiopharmaceuticals for infection imaging: mechanisms and applications. Semin Nucl Med. 2009 Jan;39(1):2-10. doi: 10.1053/j.semnuclmed.2008.08.002.

22.Gemmel F, Dumarey N, Palestro CJ. Non-invasive imaging of complicated osteomyelitis: the added value of scintigraphic techniques. Curr Radiopharm. 2008 Feb;1:73(2)-86-94. doi: 10.2174/1874471010801020073.

23.Palestro CJ, Love C, Miller TT. Diagnostic imaging tests and microbial infections. Cell Microbiol. 2007 Oct;9(10):2323-33. PMID: 17645552.

24.Kumar R, Basu S, Torigian D, Anand V, Zhuang $\mathrm{H}$, Alavi A. Role of modern imaging techniques for diagnosis of infection in the era of 18F-fluorodeoxyglucose positron emission tomography. Clin Microbiol Rev. 2008 Jan;21(1):209-24. doi: 10.1128/ CMR.00025-07.

25.De Winter F, Vogelaers D, Gemmel F, Dierckx RA. Promising role of 18-F-fluoro-Ddeoxyglucose positron emission tomography in clinical infectious diseases. Eur J Clin Microbiol Infect Dis. 2002 Apr;21(4):247-57. PMID: 12072934.

26.Keidar Z, Militianu D, Melamed E, Bar- 
Shalom R, Israel O. The diabetic foot:initial experience with 18F-FDG PET/CT. J Nucl Med. 2005 Mar;46(3):444-9. PMID: 15750157.

27. Rosen RS, Fayad L, Wahl RL. Increased
18F-FDG uptake in degenerative disease of the spine: characterization with 18F-FDG PET/CT. J Nucl Med. 2006 Aug;47(8):127480. PMID: 16883005.

\section{Correspondence:}

Ítalo Medeiros Azevedo

Avenida Nilo Peçanha, 620

59012-300 Natal-RN Brasil

cirurgex.ufrn@gmail.com

Received: Nov 19, 2016

Review: Jan 16, 2017

Accepted: Feb 20, 2017
Conflict of interest: none

Financial source: CNPq (Grant 4449083/20144)

${ }^{1}$ Research performed at Nucleus of Experimental Surgery, Department of Surgery, Universidade Federal do Rio Grande do Norte (UFRN), Natal-RN, Brazil. Part of PhD degree thesis, Postgraduate Program in Health Sciences. Tutor: Aldo Cunha Medeiros. 